J. Korean Math. Soc. 51 (2014), No. 2, pp. 363-382

http://dx.doi.org/10.4134/JKMS.2014.51.2.363

\title{
HEREDITARILY HYPERCYCLICITY AND SUPERCYCLICITY OF WEIGHTED SHIFTS
}

\author{
Yu-Xia Liang And Ze-Hua Zhou
}

\begin{abstract}
In this paper we first characterize the hereditarily hypercyclicity of the unilateral (or bilateral) weighted shifts on the spaces $L^{2}(\mathbb{N}, \mathcal{K})\left(\right.$ or $\left.L^{2}(\mathbb{Z}, \mathcal{K})\right)$ with weight sequence $\left\{A_{n}\right\}$ of positive invertible diagonal operators on a separable complex Hilbert space $\mathcal{K}$. Then we give the necessary and sufficient conditions for the supercyclicity of those weighted shifts, which extends some previous results of H. Salas. At last, we give some conditions for the supercyclicity of three different weighted shifts.
\end{abstract}

\section{Introduction}

Let $L(X)$ denote the space of linear continuous operators on a separable infinite dimensional Fréchet space $X$. For $n \in \mathbb{N}$ the set of all positive integral numbers, the $n$-th iterate of $T \in L(X)$ denoted by $T^{n}$, is the function obtained by composing $T$ with itself $n$ times. A continuous linear operator $T$ on a topological vector space $X$ is called hypercyclic (respectively, supercyclic) provided there is some $f \in X$ such that the orbit $\operatorname{Orb}(T, f)=\left\{T^{n} f: n=0,1,2, \ldots\right\}$ (respectively, the projective orbit $\left\{\lambda T^{n} f: \lambda \in \mathbb{C}, n=0,1,2, \ldots\right\}$ ) is dense in $X$. Such a vector $f$ is said to hypercyclic (respectively, supercyclic) for $T$. For motivation, examples and background about linear dynamics we refer the readers to the books [1] by Bayart and Matheron, [6] by Grosse-Erdmann and Manguillot, and article by Godefroy and Shapiro [4]. For a subsequence $\left(n_{k}\right) \subset \mathbb{N}$, we will always refer to an increasing sequence of positive integers.

Definition 1.1. Let $T \in L(X)$ and $\left(m_{k}\right)$ be a sequence of non-negative integers. We say that $T$ is hereditarily hypercyclic with respect to $\left(m_{k}\right)$ provided that the sequence $\left\{T^{m_{k_{j}}}\right\}_{j \geq 1}$ is hypercyclic for all subsequences $\left(m_{k_{j}}\right)$ of $\left(m_{k}\right)$. An operator $T$ will be called hereditarily hypercyclic if it is hereditarily hypercyclic with respect to some sequence $\left(m_{k}\right)$.

Received July 27, 2013; Revised August 19, 2013.

2010 Mathematics Subject Classification. 47A16, 47B37.

Key words and phrases. hereditarily hypercyclic, supercyclic, weighted shifts.

The second author is corresponding author. The work was supported in part by the National Natural Science Foundation of China (Grant Nos. 11371276; 11301373; 11201331; 10971153). 
Remark 1.2. The sequence $\left(m_{k}\right)$ in Definition 1.1 need not be the entire sequence $\left(m_{k}\right)=(k)$ of positive integers.

The following criterion plays an important role in the proof of the hereditarily hypercyclicity of weighted shifts.

Theorem 1.3 ([6, Theorem 3.12], Hypercyclicity Criterion). Let $T$ be an operator. If there are dense subsets $X_{0}, Y_{0} \subset X$, an increasing sequence $\left(n_{k}\right)$ of positive integers, and maps $S_{n_{k}}: Y_{0} \rightarrow X, k \geq 1$, such that, for any $x \in X_{0}, y \in Y_{0}$,

$$
T^{n_{k}} x \underset{k \rightarrow \infty}{\rightarrow} 0, \quad S_{n_{k}} y \underset{k \rightarrow \infty}{\rightarrow} 0, T^{n_{k}} S_{n_{k}} y \underset{k \rightarrow \infty}{\rightarrow} y,
$$

then we say that $T$ satisfies the Hypercyclicity Criterion with respect to $\left(n_{k}\right)$, in particular, $T$ is hypercyclic.

Also, the following Supercyclicity Criterion plays an important role in showing the supercyclicity of $T \in L(X)$.

Theorem 1.4 ([1, Definition 1.13], Supercyclicity Criterion). Let $T$ be an operator on the space $X$. If there exist a positive integer sequence $n_{k} \rightarrow \infty$ and two dense sets $X_{0}$ and $Y_{0}$ such that:

(1) there exists a map $S: Y_{0} \rightarrow X$ such that $T S z=z$ for all $z \in Y_{0}$, and

(2) if $y \in X_{0}$ and $z \in Y_{0}$, then $\left\|T^{n_{k}} y\right\|\left\|S_{n_{k}} z\right\| \rightarrow 0$ as $k \rightarrow \infty$, then $T$ is supercyclic on the space $X$.

Remark 1.5 ([1, Theorem 1.12]). If $T$ is supercyclic, then the set of supercyclic vectors of $T, S C(T)$, is a dense $G_{\delta}$ subset of $X$.

Let $\mathbb{Z}$ be the set of all integral numbers. Let $\mathcal{K}$ be a separable complex Hilbert space with an orthonormal basis $\left\{f_{k}\right\}_{k=0}^{\infty}$. Define two separable spaces as follows:

$$
\begin{aligned}
& L^{2}(\mathbb{Z}, \mathcal{K}):=\left\{x=\left(\ldots, x_{-1},\left[x_{0}\right], x_{1}, \ldots\right): x_{i} \in \mathcal{K} \text { and } \sum_{i \in \mathbb{Z}}\left\|x_{i}\right\|^{2}<\infty\right\}, \\
& L^{2}(\mathbb{N}, \mathcal{K}):=\left\{x=\left(\left[x_{1}\right], x_{2}, \ldots\right): x_{i} \in \mathcal{K} \text { and } \sum_{i \in \mathbb{N}}\left\|x_{i}\right\|^{2}<\infty\right\}
\end{aligned}
$$

Now, let $\left\{A_{n}\right\}(n \in \mathbb{Z}$ or $n \in \mathbb{N})$ be a uniformly bounded sequence of invertible positive diagonal operators on $\mathcal{K}$. For the unilateral case on $L^{2}(\mathbb{N}, \mathcal{K})$, it is known that the forward unilateral weighted shifts are never hypercyclic. Thus we need only consider that $T$ is the backward unilateral weighted shift on $L^{2}(\mathbb{N}, \mathcal{K})$.

$$
T\left(\left[x_{1}\right], x_{2}, \ldots\right)=\left(\left[A_{1} x_{2}\right], A_{2} x_{3}, \ldots\right) \text { for } x=\left(x_{j}\right)_{j \in \mathbb{N}} \in L^{2}(\mathbb{N}, \mathcal{K}) .
$$

Since $\left\{A_{n}\right\}_{n=1}^{\infty}$ is uniformly bounded, then $\|T\|=\sup _{n \in \mathbb{N}}\left\|A_{n}\right\|<\infty$. Now, for $n \in \mathbb{N}$,

$$
T^{n}\left(\left[x_{1}\right], x_{2}, \ldots\right)=\left(\left[y_{1}\right], y_{2}, \ldots\right), \text { where } y_{j}=\prod_{s=0}^{n-1} A_{j+s} x_{n+j}
$$


so that

$$
\left\|T^{n}\right\|=\sup _{j}\left\|\prod_{s=0}^{n-1} A_{j+s}\right\| .
$$

If $\left\{A_{n}^{-1}\right\}_{n=1}^{\infty}$ is also uniformly bounded, then $T^{-1}$ is the forward unilateral weighted shift on $L^{2}(\mathbb{N}, \mathcal{K})$, given by

$$
T^{-1}\left(\left[x_{1}\right], x_{2}, \ldots\right)=\left([0], A_{1}^{-1} x_{1}, A_{2}^{-1} x_{2}, \ldots\right) \text { for } x=\left(x_{j}\right)_{j \in \mathbb{N}} \in L^{2}(\mathbb{N}, \mathcal{K}) .
$$

For $n \in \mathbb{N}$,

$$
T^{-n}\left(\left[x_{1}\right], x_{2}, \ldots\right)=\left(\left[z_{1}\right], z_{2}, \ldots\right),
$$

where $z_{1}=\cdots=z_{n}=0, z_{n+j}=\prod_{s=0}^{n-1} A_{j+s}^{-1} x_{j}$, so that

$$
\left\|T^{-n}\right\|=\sup _{j}\left\|\prod_{s=0}^{n-1} A_{j+s}^{-1}\right\| .
$$

For the bilateral case on $L^{2}(\mathbb{Z}, \mathcal{K})$, we firstly let $T$ be the forward bilateral operator weighted shift on $L^{2}(\mathbb{Z}, \mathcal{K})$. For $x=\left(x_{j}\right)_{j \in \mathbb{Z}} \in L^{2}(\mathbb{Z}, \mathcal{K})$,

$$
T\left(\ldots, x_{-1},\left[x_{0}\right], x_{1}, \ldots\right)=\left(\ldots, A_{-2} x_{-2},\left[A_{-1} x_{-1}\right], A_{0} x_{0}, \ldots\right) .
$$

Also, since $\left\{A_{n}\right\}_{n=-\infty}^{\infty}$ is uniformly bounded, then $\|T\|=\sup _{i \in \mathbb{Z}}\left\|A_{i}\right\|<\infty$. For $n \in \mathbb{N}$,

$$
T^{n}\left(\ldots, x_{-1},\left[x_{0}\right], x_{1}, \ldots\right)=\left(\ldots, y_{-1},\left[y_{0}\right], y_{1}, \ldots\right)
$$

where

$$
y_{n+j}=\prod_{s=0}^{n-1} A_{j+s} x_{j} \text { or } y_{j}=\prod_{s=0}^{n-1} A_{j+s-n} x_{j-n}
$$

so that

$$
\left\|T^{n}\right\|=\sup _{j}\left\|\prod_{s=0}^{n-1} A_{j+s}\right\| .
$$

If $\left\{A_{n}^{-1}\right\}_{n=-\infty}^{\infty}$ is also uniformly bounded, then $T^{-1}$ is the backward bilateral operator weighted shift on $L^{2}(\mathbb{Z}, \mathcal{K})$, given by

$$
T^{-1}\left(\ldots, x_{-1},\left[x_{0}\right], x_{1}, \ldots\right)=\left(\ldots, A_{-1}^{-1} x_{0},\left[A_{0}^{-1} x_{1}\right], A_{1}^{-1} x_{2} \ldots\right) .
$$

For $n \in \mathbb{N}$,

$$
T^{-n}\left(\ldots, x_{-1},\left[x_{0}\right], x_{1}, \ldots\right)=\left(\ldots, z_{-1},\left[z_{0}\right], z_{1}, \ldots\right),
$$

where

$$
z_{j-n}=\prod_{s=0}^{n-1} A_{j-n+s}^{-1} x_{j} \text { or } z_{j}=\prod_{s=0}^{n-1} A_{j+s}^{-1} x_{n+j},
$$


so that

$$
\left\|T^{-n}\right\|=\sup _{j}\left\|\prod_{s=0}^{n-1} A_{j-n+s}^{-1}\right\|=\sup _{j}\left\|\prod_{s=j-n}^{j-1} A_{s}^{-1}\right\| .
$$

Secondly, let $T$ be the backward bilateral shift on $L^{2}(\mathbb{Z}, \mathcal{K})$, then

$$
T^{n}\left(\ldots, x_{-1},\left[x_{0}\right], x_{1}, \ldots\right)=\left(\ldots, y_{-1},\left[y_{0}\right], y_{1}, \ldots\right)
$$

where

$$
y_{j}=\prod_{s=0}^{n-1} A_{j+s} x_{n+j} \text { or } y_{j-n}=\prod_{s=0}^{n-1} A_{j-n+s} x_{j}
$$

so that

$$
\left\|T^{n}\right\|=\sup _{j}\left\|\prod_{s=0}^{n-1} A_{j-n+s}\right\|=\sup _{j}\left\|\prod_{s=j-n}^{j-1} A_{s}\right\| .
$$

Further,

$$
T^{-n}\left(\ldots, x_{-1},\left[x_{0}\right], x_{1}, \ldots\right)=\left(\ldots, z_{-1},\left[z_{0}\right], z_{1}, \ldots\right)
$$

where

$$
z_{j}=\prod_{s=0}^{n-1} A_{j+s-n}^{-1} x_{j-n} \text { or } z_{n+j}=\prod_{s=0}^{n-1} A_{j+s}^{-1} x_{j}
$$

so that

$$
\left\|T^{-n}\right\|=\sup _{j}\left\|\prod_{s=0}^{n-1} A_{j+s}^{-1}\right\| .
$$

Since each $A_{n}$ is an invertible diagonal operator on $\mathcal{K}$, we have that

$$
\begin{aligned}
& \left\|A_{n}\right\|=\sup _{k}\left\|A_{n} f_{k}\right\|, \quad\left\|A_{n}^{-1}\right\|=\sup _{k}\left\|A_{n}^{-1} f_{k}\right\| \text { and } \\
& \sup _{k}\left\|A_{n} f_{k}\right\|=\frac{1}{\inf _{k}\left\|A_{n}^{-1} f_{k}\right\|} .
\end{aligned}
$$

In the following, for $n \geq 0$ and $q \in \mathbb{N}$, denote $H_{q, n}=\left\{x=\left(x_{i}\right) \in L^{2}(\mathbb{N}, \mathcal{K})\right.$ : $\left.x_{i}=0, \forall i+n>q\right\}$ dense subsets of $L^{2}(\mathbb{N}, \mathcal{K})$ and $G_{q, n}=\left\{x=\left(x_{i}\right) \in\right.$ $\left.L^{2}(\mathbb{Z}, \mathcal{K}): x_{i}=0, \forall|i+n|>q\right\}$ dense subsets of $L^{2}(\mathbb{Z}, \mathcal{K})$. Moreover, for $x \in \mathcal{K}$ and $i \in \mathbb{N}$, let $x(i):=\left(\left[y_{1}\right], y_{2}, \ldots\right)$ in $L^{2}(\mathbb{N}, \mathcal{K})$, where $y_{i}=x$ and $y_{j}=0$ for all $j \neq i$.

This article is organized as follows: we will follow and complement the work of [7], to determine those weighted shifts that are hereditarily hypercyclic with respect to a given sequence $\left(n_{k}\right) \subset \mathbb{N}$ (Theorem 2.5 and Theorem 2.6) in Section 2 . Then we give the necessary and sufficient conditions for the supercyclicity of those weighted shifts in Section 3, which extends some previous results of $H$. Salas [11]. At last, we characterize the supercyclicity of $B$ on the space $L^{p}(\beta)$ 
and weighted pseudo-shifts on an arbitrary $F$-space in which the canonical unit vectors $\left(e_{n}\right)$ form a Schauder basis in Section 4 and Section 5, respectively.

\section{Hereditarily hypercyclic on the class of weighted shifts}

This part is inspired by the recent paper [7, Theorem 3.1 and Theorem 3.3], in which the authors gave some characterizations for the bilateral weighted shift $T$ to be hypercyclic. Now we will show the hereditarily hypercyclicity of the unilateral and bilateral weighted shifts, respectively.

Proposition 2.1 ([2, Theorem 2.3]). Let $T \in L(X)$. The following are equivalent:

(1) T satisfies the Hypercyclicity Criterion.

(2) $T$ is hereditarily hypercyclic.

(3) $T \bigoplus T$ is hypercyclic.

Remark 2.2. From the proof of [2, Theorem 2.3], we notice that $T$ is hereditarily hypercyclic with respect to $\left(n_{k}\right)$ whenever $T$ satisfies $(1.1)$ for $\left(n_{k}\right)$.

Theorem 2.3. Let $T$ be a unilateral weighted shift on $X=L^{2}(\mathbb{N}, \mathcal{K})$ with weight sequence $\left\{A_{n}\right\}_{n=1}^{\infty}$, where $\left\{A_{n}\right\}$ is a uniformly bounded sequence of positive invertible diagonal operators on $\mathcal{K}$. Then $\left\{T^{n_{k}}\right\}_{k>1}$ is hypercyclic if and only if for all $\epsilon>0$ and all $q \in \mathbb{N}$, there exists $m=m(\epsilon, q) \in\left(n_{k}\right)$ arbitrarily large satisfying

$$
\left\|\prod_{s=0}^{m-1} A_{j+s}^{-1}\right\|<\epsilon \quad(1 \leq j \leq q)
$$

Proof. $(\Leftarrow)$ Suppose that $(2.1)$ holds, we use Theorem 1.3 to show the hypercyclicity of $\left\{T^{n_{k}}\right\}_{k \geq 1}$. For $q \in \mathbb{N}$, by $(2.1)$, choose a subsequence $\left(n_{k_{q}}\right) \subset\left(n_{k}\right)$ satisfying

$$
\left\|\prod_{s=0}^{n_{k_{q}}-1} A_{j+s}^{-1}\right\|<\frac{1}{q}(1 \leq j \leq q) .
$$

Let $X_{0}=Y_{0}:=H_{q, 0}=\left\{x=\left(x_{i}\right) \in L^{2}(\mathbb{N}, \mathcal{K}): x_{i}=0, \forall i+n>q\right\}$ be dense subsets of $X$, and let $S: Y_{0} \rightarrow X$ be the mapping defined by

$$
S\left(\left[x_{1}\right], x_{2}, \ldots\right)=\left([0], A_{1}^{-1} x_{1}, A_{2}^{-1} x_{2}, \ldots\right), \quad\left(\left[x_{1}\right], x_{2}, \ldots\right) \in Y_{0} .
$$

Notice that $T S=I d_{Y_{0}}$ and $T^{n} \underset{n \rightarrow \infty}{\rightarrow} 0$ pointwise on $X_{0}$.

It suffices to show that $S^{n_{k_{q}}} \underset{q \rightarrow \infty}{\stackrel{n \rightarrow \infty}{\rightarrow}} 0$ pointwise on $Y_{0}$. For $g \in H_{q, 0}$, then $S^{n_{k_{q}}} g \in H_{q, n}$. By (1.4) and (2.2),

$$
\left\|S^{n_{k_{q}}} g\right\| \leq \sup _{1 \leq j \leq q}\left\|\prod_{s=1}^{n_{k_{q}}-1} A_{j+s}^{-1}\right\|\|g\| \leq \frac{1}{q}\|g\| \underset{q \rightarrow \infty}{\rightarrow} 0 .
$$

Thus $\left\{T^{n_{k}}\right\}_{k \geq 1}$ is hypercyclic. 
$(\Rightarrow)$ Suppose that $\left\{T^{n_{k}}\right\}_{k>1}$ is hypercyclic. Let $\epsilon>0$ and $q \in \mathbb{N}$ be given. Choose $\delta>0$ such that $\frac{\delta}{1-\delta}<\epsilon$. For arbitrary fixed nonnegative integer $i$, consider the vector $\sum_{j=1}^{q} f_{i}(j) \in L^{2}(\mathbb{N}, \mathcal{K})$. Since the set of hypercyclic vectors for $\left\{T^{n_{k}}\right\}_{k \geq 1}$ is dense in $X$, there exist a hypercyclic vector $x=\left(\left[x_{1}\right], x_{2}, \ldots\right)$ and a positive integer $n_{k}>q$ satisfying

$$
\left\|x-\sum_{j=1}^{q} f_{i}(j)\right\|<\delta \text { and }\left\|T^{n_{k}} x-\sum_{j=1}^{q} f_{i}(j)\right\|<\delta .
$$

Denote $T^{n_{k}} x=\left(\left[y_{1}\right], y_{2}, \ldots\right)$, where $y_{j}=\prod_{s=0}^{n_{k}-1} A_{j+s} x_{n_{k}+j}$. Then from the first inequality in $(2.3)$,

$$
\begin{cases}\text { (i) }\left\|x_{j}\right\|<\delta & \text { for all } j>q ; \\ \text { (ii) }\left\|x_{j}-f_{i}\right\|<\delta & \text { for all } 1 \leq j \leq q,\end{cases}
$$

since each $x_{j} \in \mathcal{K}$, there exist scalars $\alpha_{k}^{(j)}$ such that $x_{j}=\sum_{k=0}^{\infty} \alpha_{k}^{(j)} f_{k}$. So $y_{j}=\sum_{l=0}^{\infty} \alpha_{l}^{\left(j+n_{k}\right)} \prod_{s=0}^{n_{k}-1} A_{j+s} f_{l}$.

Now for $j>q,(2.4)(\mathrm{i})$ gives $\left|\alpha_{k}^{(j)}\right|<\delta$ for all $k$. For $1 \leq j \leq q$, (2.4)(ii) gives

$$
\begin{cases}\left|\alpha_{k}^{(j)}\right|<\delta, & k \neq i \\ \left|\alpha_{k}^{(j)}\right|>1-\delta, & k=i .\end{cases}
$$

For $1 \leq j \leq q$, from the second inequality in (2.3), $\left\|y_{j}-f_{i}\right\| \leq \delta$ gives

$$
\begin{cases}\left\|\alpha_{l}^{\left(j+n_{k}\right)} \prod_{s=0}^{n_{k}-1} A_{j+s} f_{l}-f_{i}\right\|<\delta, & l=i \\ \left\|\alpha_{l}^{\left(j+n_{k}\right)} \prod_{s=0}^{n_{k}-1} A_{j+s} f_{l}\right\|<\delta, & l \neq i .\end{cases}
$$

By the first inequality in (2.6) we obtain that

$$
\left\|\alpha_{l}^{\left(j+n_{k}\right)} \prod_{s=0}^{n_{k}-1} A_{j+s} f_{i}\right\|>1-\delta .
$$

Since the hypothesis $n_{k}>q$ implies $n_{k}+j>q$ for $1 \leq j \leq q$, it follows that $\left|\alpha_{l}^{\left(j+n_{k}\right)}\right|<\delta$. Thus

$$
\left\|\prod_{s=0}^{n_{k}-1} A_{j+s} f_{i}\right\|>\frac{1-\delta}{\delta}(1 \leq j \leq q) .
$$

Then

And by (1.13),

$$
\inf _{i}\left\|\prod_{s=0}^{n_{k}-1} A_{j+s} f_{i}\right\|>\frac{1-\delta}{\delta}(1 \leq j \leq q) .
$$

$$
\sup _{i}\left\|\prod_{s=0}^{n_{k}-1} A_{j+s}^{-1} f_{i}\right\|=\frac{1}{\inf _{i}\left\|\prod_{s=0}^{n_{k}-1} A_{j+s} f_{i}\right\|}<\frac{\delta}{1-\delta} \quad(1 \leq j \leq q) .
$$


From which it follows that

$$
\left\|\prod_{s=0}^{n_{k}-1} A_{j+s}^{-1}\right\|<\frac{\delta}{1-\delta}<\epsilon \quad(1 \leq j \leq q) .
$$

This completes the proof.

The following theorem is a slight modification of [7, Theorem 3.1].

Theorem 2.4. Let $T$ be a forward bilateral operator weighted shift on $L^{2}(\mathbb{Z}, \mathcal{K})$ with weight sequence $\left\{A_{n}\right\}_{n=-\infty}^{\infty}$, where $\left\{A_{n}\right\}$ is a uniformly bounded sequence of positive invertible diagonal operators on $\mathcal{K}$. Let $\left(m_{k}\right) \subset \mathbb{N}$. The the following are equivalent:

(a) $\left\{T^{m_{k}}\right\}_{k \geq 1}$ is hypercyclic.

(b) For all $\epsilon>0$ and $q \in \mathbb{N}$, there exists $m=m(\epsilon, q) \in\left(m_{k}\right)$ arbitrarily large so that for all $|j| \leq q$

$$
\left\{\begin{array}{l}
\text { (i) }\left\|\prod_{s=0}^{m-1} A_{j+s}\right\|<\epsilon, \\
\text { (ii) }\left\|\prod_{s=1}^{m} A_{j-s}^{-1}\right\|<\epsilon .
\end{array}\right.
$$

(c) T satisfies Hypercyclicity Criterion for some subsequence $\left(n_{k}\right)$ of $\left(m_{k}\right)$.

Case I: Backward unilateral weighted shift. For this case we will mainly use Theorem 2.3 .

Theorem 2.5. Let $T$ be a backward unilateral weighted shift on $L^{2}(\mathbb{N}, \mathcal{K})$ with weight sequence $\left\{A_{n}\right\}_{n=1}^{\infty}$, where $\left\{A_{n}\right\}$ is a uniformly bounded sequence of positive invertible diagonal operators on $\mathcal{K}$. Let $\left(n_{k}\right) \subset \mathbb{N}$. Then the following are equivalent:

(1) $T$ is hereditarily hypercyclic with respect to $\left(n_{k}\right)$.

(2) For all $\epsilon>0$ and $q \in \mathbb{N}$, there exists $k_{0} \in \mathbb{N}$ satisfying: for all $k \geq k_{0}$ and all $1 \leq j \leq q$,

$$
\left\|\prod_{s=0}^{n_{k}-1} A_{j+s}^{-1}\right\|<\epsilon
$$

Proof. $(2) \Rightarrow(1)$. This is obvious from Theorem 2.3 .

$(1) \Rightarrow(2)$. For a given $\epsilon>0$ and $q \in \mathbb{N}$, denote the set

$$
\mathcal{A}:=\left\{m \in\left(n_{k}\right):\left\|\prod_{s=0}^{n_{k}-1} A_{j+s}^{-1}\right\|<\epsilon \text { for all } 1 \leq j \leq q\right\} .
$$

If $\left\{n_{k}\right\} \backslash \mathcal{A}$ is infinite, then there exists a subsequence $\left(n_{k_{j}}\right) \subset\left(n_{k}\right)$ that does not satisfy (2.9). Hence by Theorem 2.3, $\left\{T^{n_{k_{j}}}\right\}_{j \geq 1}$ is not hypercyclic, it is a contradiction. This completes the proof.

Case II: Bilateral case. For this case we will mainly employ Theorem 2.4. 
Theorem 2.6. Let $T$ be a forward bilateral operator weighted shift on $L^{2}(\mathbb{Z}, \mathcal{K})$ with weight sequence $\left\{A_{n}\right\}_{n=-\infty}^{\infty}$, where $\left\{A_{n}\right\}$ is a uniformly bounded sequence of positive invertible diagonal operators on $\mathcal{K}$ and $\left\{A_{n}^{-1}\right\}$ is also a uniformly bounded sequence. We denote $M:=\max \left\{\sup _{n \in \mathbb{Z}}\left\|A_{n}\right\|, \sup _{n \in \mathbb{Z}}\left\|A_{n}^{-1}\right\|\right\}$. Let also $\left(n_{k}\right) \subset \mathbb{N}$. Then the following are equivalent:

(1) $T$ is hereditarily hypercyclic with respect to $\left(n_{k}\right)$.

(2) For all $\epsilon>0$ and $q \in \mathbb{N}$, there exists $k_{0} \in \mathbb{N}$ satisfying: for all $k \geq k_{0}$ and all $|j| \leq q$,

$$
\left\{\begin{array}{l}
\text { (i) }\left\|\prod_{s=0}^{n_{k}-1} A_{j+s}\right\|<\epsilon \\
\text { (ii) }\left\|\prod_{s=1}^{n_{k}} A_{j-s}^{-1}\right\|<\epsilon .
\end{array}\right.
$$

(3) For all $j \in \mathbb{Z}$,

$$
\lim _{k \rightarrow \infty}\left\|\prod_{s=0}^{j+n_{k}-1} A_{s}\right\|=0
$$

and

$$
\lim _{k \rightarrow \infty}\left\|\prod_{s=1}^{j+n_{k}} A_{-s}^{-1}\right\|=0
$$

Proof. $(1) \Rightarrow(2)$. Given $\epsilon>0$ and $q \in \mathbb{N}$, let the set

$$
\mathcal{A}:=\left\{m \in\left(n_{k}\right):\left\{\begin{array}{l}
\text { (i) }\left\|\prod_{s=0}^{n_{k}-1} A_{j+s}\right\|<\epsilon, \\
\text { (ii) }\left\|\prod_{s=1}^{n_{k}} A_{j-s}^{-1}\right\|<\epsilon,
\end{array} \quad \text { for all }|j| \leq q\right\} .\right.
$$

If $\left(n_{k}\right) \backslash \mathcal{A}$ is infinite, then there exists a subsequence $\left(n_{k_{j}}\right) \subset\left(n_{k}\right) \backslash \mathcal{A}$ that does not satisfy (2.10). Hence by Theorem $2.4,\left\{T^{n_{k_{j}}}\right\}_{j \geq 1}$ is not hypercyclic, which is a contradiction.

$(2) \Rightarrow(1)$. This follows from Theorem 2.4.

$(2) \Rightarrow(3)$. Fix $j \in \mathbb{Z}$. On the one hand, if $j \geq 1$, then by (2.10)(i) and (ii),

$$
\left\|\prod_{s=0}^{j+n_{k}-1} A_{s}\right\| \leq\left\|A_{0} A_{1} \ldots A_{j-1}\right\|\left\|A_{j} A_{j+1} \ldots A_{j+n_{k}-1}\right\| \leq M^{j} \epsilon
$$

and

$$
\left\|\prod_{s=1}^{j+n_{k}} A_{-s}^{-1}\right\| \leq\left\|A_{-1}^{-1} A_{-2}^{-1} \ldots A_{-j}^{-1}\right\|\left\|A_{-j-1}^{-1} A_{-j-2}^{-1} \ldots A_{-\left(j+n_{k}\right)}^{-1}\right\| \leq M^{j} \epsilon .
$$

On the other hand, if $j<0$, we have that

$$
\left\|\prod_{s=0}^{j+n_{k}-1} A_{s}\right\| \leq\left\|A_{j}^{-1} \ldots A_{-1}^{-1}\right\|\left\|A_{j} \ldots A_{0} \ldots A_{j+n_{k}-1}\right\| \leq M^{j} \epsilon
$$

and

$$
\left\|\prod_{s=1}^{j+n_{k}} A_{-s}^{-1}\right\| \leq\left\|A_{-j-1} \ldots A_{0}\right\|\left\|A_{-j-1}^{-1} \ldots A_{0}^{-1} A_{-1}^{-1} \ldots A_{-j-n_{k}}^{-1}\right\| \leq M^{j} \epsilon .
$$


From the above four inequality it follows (3).

$(3) \Rightarrow(2)$. For $\epsilon>0$ and $q \in \mathbb{N}$, let

$$
\delta:=\max \left\{\left\|A_{0}^{-1} \ldots A_{j-1}^{-1}\right\|,\left\|A_{-1} \ldots A_{-j}\right\|, M^{2 q}: 1 \leq j \leq q\right\} .
$$

From (2.11) and (2.12), choose $k_{0}$ such that for all $|j| \leq q$ and $k \geq k_{0}$, we have that

$$
\left\{\begin{array}{l}
\text { (i) }\left\|\prod_{s=0}^{j+n_{k}-1} A_{s}\right\|<\frac{\epsilon}{\delta}, \\
\text { (ii) }\left\|\prod_{s=1}^{j+n_{k}} A_{-s}^{-1}\right\|<\frac{\epsilon}{\delta} .
\end{array}\right.
$$

Hence, for $k \geq k_{0}$, by (2.13) it follows that

$$
\begin{aligned}
\left\|\prod_{s=0}^{n_{k}-1} A_{s+j}\right\| & \leq \begin{cases}\left\|A_{0} \ldots A_{j+n_{k}-1}\right\|\left\|A_{0}^{-1} \ldots A_{j-1}^{-1}\right\|, & j>0 \\
\left\|A_{0} A_{1} \ldots A_{j+n_{k}-1}\right\|, & j=0 \\
\left\|A_{j} A_{j+1} \ldots A_{-1}\right\|\left\|A_{0} \ldots A_{j+n_{k}-1}\right\|, & j<0\end{cases} \\
& \leq \begin{cases}\frac{\epsilon}{\delta} \delta=\epsilon, & j>0, \\
\frac{\epsilon}{\delta}, & j=0, \\
\delta \frac{\epsilon}{\delta}=\epsilon, & j<0 .\end{cases}
\end{aligned}
$$

At the same time

$$
\begin{aligned}
\left\|\prod_{s=1}^{n_{k}} A_{j-s}^{-1}\right\| \leq & \begin{cases}\left\|A_{-1} \ldots A_{j}\right\|\left\|A_{-1}^{-1} \ldots A_{j-n_{k}}^{-1}\right\|, & j<0, \\
\left\|A_{-1}^{-1} \ldots A_{j-n_{k}}^{-1}\right\|, & j=0, \\
\left\|A_{j-1}^{-1} \ldots A_{0}^{-1}\right\|\left\|A_{-1}^{-1} \ldots A_{j-n_{k}}^{-1}\right\|, & j>0,\end{cases} \\
& \leq \begin{cases}\delta \frac{\epsilon}{\delta}=\epsilon, \quad j>0, \\
\frac{\epsilon}{\delta}, \quad j=0, \\
\delta \frac{\epsilon}{\delta}=\epsilon, \quad j<0 .\end{cases}
\end{aligned}
$$

This completes the proof.

Now for a backward bilateral operator weighted shift on $L^{2}(\mathbb{Z}, \mathcal{K})$, a similar result can be stated as follows:

Theorem 2.7. Let $T$ be a backward bilateral operator weighted shift on $L^{2}(\mathbb{Z}$, $\mathcal{K})$ with weight sequence $\left\{A_{n}\right\}_{n=-\infty}^{\infty}$, where $\left\{A_{n}\right\}$ is a uniformly bounded sequence of positive invertible diagonal operators on $\mathcal{K}$ and $\left\{A_{n}^{-1}\right\}$ is also a uniformly bounded sequence. We denote $M:=\max \left\{\sup _{n \in \mathbb{Z}}\left\|A_{n}\right\|, \sup _{n \in \mathbb{Z}}\left\|A_{n}^{-1}\right\|\right\}$. Let also $\left(n_{k}\right) \subset \mathbb{N}$. Then the following are equivalent:

(1) $T$ is hereditarily hypercyclic with respect to $\left(n_{k}\right)$.

(2) For all $\epsilon>0$ and $q \in \mathbb{N}$, there exists $k_{0} \in \mathbb{N}$ satisfying: for all $k \geq k_{0}$ and all $|j| \leq q$,

$$
\left\{\begin{array}{l}
\text { (i) }\left\|\prod_{s=0}^{n_{k}-1} A_{j+s}^{-1}\right\|<\epsilon, \\
\text { (ii) }\left\|\prod_{s=1}^{n_{k}} A_{j-s}\right\|<\epsilon .
\end{array}\right.
$$


(3) For all $j \in \mathbb{Z}$,

$$
\lim _{k \rightarrow \infty}\left\|\prod_{s=0}^{j+n_{k}-1} A_{s}^{-1}\right\|=0 \text { and } \lim _{k \rightarrow \infty}\left\|\prod_{s=1}^{j+n_{k}} A_{-s}\right\|=0 .
$$

Remark 2.8. If $T$ is bilateral weighted shift on $L^{2}(\mathbb{Z}, \mathcal{K})$ with weight sequence $\left\{A_{n}\right\}_{n=-\infty}^{\infty}$, then $T$ is invertible if and only if there exists $M>0$ such that $\left\|A_{n}^{-1}\right\| \leq M$ for all $n \in \mathbb{Z}$. Thus the operator $T$ defined in Theorem 2.6 (or Theorem 2.7) is invertible.

\section{Supercyclic weighted shift}

In [11] Salas provided a characterization for supercyclic bilateral weighted shifts acting on $l^{p}(\mathbb{Z})$ with $p \geq 1$ or $c_{0}(\mathbb{Z})$ with positive weight sequence $\left\{w_{n}\right\}$, in terms of their weight sequences as follows:

Proposition 3.1 ([11, Theorem 3.1]). Let $T$ be a bilateral backward weighted shift, acting on $l^{p}(\mathbb{Z})$ with $p \geq 1$ or $c_{0}(\mathbb{Z})$ with positive weight sequence $\left\{w_{n}\right\}$; i.e., $T e_{n}=w_{n} e_{n-1}$ for $n \in \mathbb{Z}$. The operator $T$ is supercyclic if and only if

$$
\liminf _{n \rightarrow \infty} \max \left\{\frac{\prod_{j+1-n \leq k \leq j} w_{k}}{\prod_{h+1 \leq k \leq h+n} w_{k}}:|j|,|h| \leq q\right\}=0
$$

for all $q \in \mathbb{N}$.

The characterization of Salas turns out to be a particular case of Theorem 3.3 when $\operatorname{dim} \mathcal{K}=1$.

Theorem 3.2. Let $T$ be a forward bilateral operator weighted shift on $X=$ $L^{2}(\mathbb{Z}, \mathcal{K})$ with weight sequence $\left\{A_{n}\right\}_{n=-\infty}^{\infty}$, where $\left\{A_{n}\right\}$ is a uniformly bounded sequence of positive invertible diagonal operators on $\mathcal{K}$. Then $T$ is supercyclic if and only if for every $q \in \mathbb{N}$,

$$
\liminf _{n \rightarrow \infty} \max \left\{\left\|\prod_{k=j}^{j+n-1} A_{k}\right\|\left\|\prod_{k=h-n}^{h-1} A_{k}^{-1}\right\|:|j|,|h| \leq q\right\}=0 .
$$

Proof. Suppose $T$ is supercyclic. Let $\epsilon>0, q \in \mathbb{N}$ be given; choose $\delta>0$ such that $\frac{\delta}{1-\delta}<\epsilon$. For arbitrary fixed non-negative integer $i$, consider the vector $\sum_{|j| \leq q} f_{i}(j) \in L^{2}(\mathbb{Z}, \mathcal{K})$. By Remark 1.5, there exists a supercyclic vector $x=\left(\ldots, x_{-1},\left[x_{0}\right], x_{1}, \ldots\right)$ such that

$$
\left\|x-\sum_{|j| \leq q} f_{i}(j)\right\|<\delta .
$$

Then it follows that

$$
\begin{cases}\left\|x_{j}\right\|<\delta & \text { for all }|j|>q \\ \left\|x_{j}-f_{i}\right\|<\delta & \text { for all }|j| \leq q .\end{cases}
$$


Since $x \in L^{2}(\mathbb{Z}, \mathcal{K})$, there exists scalars $\alpha_{k}^{(j)}$ such that $x_{j}=\sum_{k=0}^{\infty} \alpha_{k}^{(j)} f_{k}$. From $(3.2)$,

$$
\begin{cases}\left|\alpha_{k}^{(j)}\right|<\delta, & |j|>q \text { and for all } k \\ \left|\alpha_{k}^{(j)}\right|<\delta, & |j| \leq q \text { and for } k \neq i \\ \left|\alpha_{k}^{(j)}\right|>1-\delta, & |j| \leq q \text { and for } k=i\end{cases}
$$

On the other hand, since $\mathbb{C O r b}(T, x)$ is dense in $L^{2}(\mathbb{Z}, \mathcal{K})$, there exist a positive integer $n>2 q$ and $0 \neq \alpha \in \mathbb{C}$ such that

$$
\left\|\alpha T^{n} x-\sum_{|j| \leq q} f_{i}(j)\right\|<\delta
$$

where $T^{n} x=\left(\ldots, y_{-1},\left[y_{0}\right], y_{1}, \ldots\right)$. Then,

$$
\begin{cases}\left\|\alpha y_{j+n}\right\|<\delta, & \text { if }|j+n|>q \\ \left\|\alpha y_{j+n}-f_{i}\right\|<\delta, & \text { if }|j+n| \leq q .\end{cases}
$$

From the hypothesis $n>2 q$, we have that $j+n>q$ for all $|j| \leq q$ and hence it follows that

$$
\left\|\alpha y_{j+n}\right\|<\delta \text { for all }|j| \leq q
$$

By (1.5),

$$
y_{j+n}=\prod_{s=0}^{n-1} A_{j+s} x_{j}=\sum_{k=0}^{\infty} \alpha_{k}^{(j)} \prod_{s=0}^{n-1} A_{j+s} f_{k}
$$

That is,

$$
\left|\alpha\left\|\alpha_{k}^{(j)} \mid\right\| \prod_{s=0}^{n-1} A_{j+s} f_{k} \|<\delta \text { for all } k \text { and }\right| j \mid \leq q .
$$

In particular, if $k=i$, it follows that

$$
|\alpha|\left\|\prod_{s=0}^{n-1} A_{j+s} f_{i}\right\|<\frac{\delta}{1-\delta} \text { for all }|j| \leq q .
$$

Thus

$$
\left\|\prod_{s=j}^{j+n-1} A_{s}\right\|=\sup _{i}\left\|\prod_{s=0}^{n-1} A_{j+s} f_{i}\right\|<\frac{\delta}{|\alpha|(1-\delta)} \quad \text { for all }|j| \leq q .
$$

Again from (1.5) it follows that

$$
y_{j}=\prod_{s=0}^{n-1} A_{j+s-n} x_{j-n}=\sum_{k=0}^{\infty} \alpha_{k}^{(j-n)} \prod_{s=0}^{n-1} A_{j+s-n} f_{k} .
$$

Then from (3.4) we obtain that

$$
\begin{cases}\text { (i) }\left\|\alpha \alpha_{k}^{(j-n)} \prod_{s=0}^{n-1} A_{j+s-n} f_{k}-f_{k}\right\|<\delta & \text { for } k=i \\ \text { (ii) }\left\|\alpha \alpha_{k}^{(j-n)} \prod_{s=0}^{n-1} A_{j+s-n} f_{k}\right\|<\delta & \text { for } k \neq i\end{cases}
$$


Since $n>2 q$, it follows that $|j-n|>q$ for all $|j| \leq q$. Hence from (3.3) we obtain that $\left|\alpha_{k}^{(j-n)}\right|<\delta$ for all $k$. In particular,

$$
\left|\alpha_{i}^{(j-n)}\right|<\delta \text { for all }|j| \leq q .
$$

Hence from (3.5)(i) we obtain that

$$
|\alpha|\left|\alpha_{i}^{(j-n)}\right|\left\|\prod_{s=0}^{n-1} A_{j+s-n} f_{i}\right\|>1-\delta \text { for all }|j| \leq q .
$$

Thus combining (3.6) and (3.7) it follows that

$$
|\alpha|\left\|\prod_{k=j-n}^{j-1} A_{k} f_{i}\right\|>\frac{\delta}{1-\delta} \text { for all }|j| \leq q .
$$

Since the above inequality holds for arbitrary $i$, it follows that

$$
\inf _{i}\left\|\prod_{k=j-n}^{j-1} A_{k} f_{i}\right\|>\frac{1-\delta}{|\alpha| \delta} \text { for all }|j| \leq q,
$$

and so

$$
\sup _{i}\left\|\prod_{k=j-n}^{j-1} A_{k}^{-1} f_{i}\right\|=\frac{1}{\inf _{i}\left\|\prod_{k=j-n}^{j-1} A_{k} f_{i}\right\|}<\frac{|\alpha| \delta}{1-\delta} \text { for all }|j| \leq q .
$$

Therefore

$$
\left\|\prod_{k=j-n}^{j-1} A_{k}^{-1}\right\|<\frac{|\alpha| \delta}{1-\delta} \text { for all }|j| \leq q .
$$

Consequently, combining (3) with (3.8),

$$
\left\|\prod_{k=j}^{j+n-1} A_{k}\right\|\left\|\prod_{s=h-n}^{h-1} A_{s}^{-1}\right\|<\frac{\delta}{|\alpha|(1-\delta)} \frac{|\alpha| \delta}{1-\delta}<\epsilon^{2} \quad \text { for all }|h|,|j| \leq q .
$$

That is, (3.1) holds.

Conversely, suppose (3.1) holds, we use Theorem 1.4 to prove the sufficiency. Let $q \in \mathbb{N}$ and $X_{0}=Y_{0}:=G_{q, 0}$ be dense subsets of $L^{2}(\mathbb{Z}, \mathcal{K})$. For any $g, h \in G_{q, 0}$, it follows that $T^{n} h \in G_{q,-n}$ and $T^{-n} g \in G_{q, n}$. Define the mapping $S: Y_{0} \rightarrow X$ by

$$
S\left(\ldots, x_{-1},\left[x_{0}\right], x_{1}, \ldots\right)=\left(\ldots, A_{-1}^{-1} x_{0},\left[A_{0}^{-1} x_{1}\right], A_{1}^{-1} x_{2}, \ldots\right),
$$

and let $S_{n_{k}}=S^{n_{k}}$. It is obvious that

$$
T S=I d_{Y_{0}} .
$$

Moreover, by (1.6) and (1.8), we get that

$$
\left\|T^{n} g\right\| \leq \max \left\{\left\|\prod_{k=j}^{j+n-1} A_{k}\right\|, \quad|j| \leq q\right\}\|g\|,
$$




$$
\left\|S_{n} h\right\| \leq \max \left\{\left\|\prod_{k=h-n}^{h-1} A_{k}^{-1}\right\|:|h| \leq q\right\}\|h\| .
$$

Thus it follows that

$$
\left\|T^{n} g\right\|\left\|S_{n} h\right\| \leq \max \left\{\left\|\prod_{k=j}^{j+n-1} A_{k}\right\|\left\|\prod_{k=h-n}^{h-1} A_{k}^{-1}\right\|:|j| \leq q,|h| \leq q\right\}\|g\|\|h\| .
$$

By (3.1) there exist a sequence $\left(n_{k}\right)$ such that

$$
\lim _{k \rightarrow \infty} \max \left\{\left\|\prod_{s=j}^{j+n_{k}-1} A_{s}\right\|\left\|\prod_{s=h-n_{k}}^{h-1} A_{s}^{-1}\right\|:|j| \leq q,|h| \leq q\right\}\|x\|\|y\|=0,
$$

and consequently

$$
\lim _{k \rightarrow \infty}\left\|T^{n_{k}}(x)\right\|\left\|S_{n_{k}}(y)\right\|=0 .
$$

From (3.9) and (3.10) we obtain the sufficiency. The proof is complete.

Now for a backward bilateral operator weighted shift on $L^{2}(\mathbb{Z}, \mathcal{K})$, a similar result can be stated as follows:

Theorem 3.3. Let $T$ be a backward bilateral operator weighted shift on $L^{2}(\mathbb{Z}$, $\mathcal{K})$ with weight sequence $\left\{A_{n}\right\}_{n=-\infty}^{\infty}$ where $\left\{A_{n}\right\}$ is a uniformly bounded sequence of positive invertible diagonal operator on $\mathcal{K}$. Then $T$ is supercyclic if and only if for every $q \in \mathbb{N}$,

$$
\liminf _{n \rightarrow \infty} \max \left\{\left\|\prod_{k=j-1}^{j-n} A_{k}\right\|\left\|\prod_{k=h}^{h+n-1} A_{k}^{-1}\right\|:|j|,|h| \leq q\right\}=0
$$

\section{Supercyclicity of $B$ on $L^{p}(\beta)$}

For a sequence of positive numbers $\{\beta(n)\}_{n=-\infty}^{\infty}$ with $\beta(0)=1$ and $1 \leq p<$ $\infty$, the space of formal Laurent series consists of the sequences $f=\{\hat{f}(n)\}_{n=-\infty}^{\infty}$ such that

$$
\|f\|^{p}=\|f\|_{\beta}^{p}=\sum_{n=-\infty}^{\infty}|\hat{f}(n)|^{p} \beta(n)^{p}<\infty .
$$

We will use the notation $f(z)=\sum_{n=-\infty}^{\infty} \hat{f}(n) z^{n}$ whether or not the series converges for any $z$. These are called formal Laurent series. As we all know, they are called formal power series and are denoted by $H^{p}(\beta)$, when $n$ ranges on $\mathbb{N} \cup\{0\}$.

Now, let $L^{p}(\beta)$ denotes a reflexive Banach space with the norm $\|\cdot\|_{\beta}$. Let $\hat{f}_{k}(n)=\delta_{k}(n)$. So $f_{k}(z)=z^{k}$ and the $\left\{f_{k}\right\}_{k \in \mathbb{Z}}$ is a basis for $L^{p}(\beta)$ such that $\left\|f_{k}\right\|=\beta(k)$. The operator $B$ on $L^{p}(\beta)$ is defined by

$$
B f_{j}=f_{j-1}, \quad j \in \mathbb{Z} \text {. }
$$


Clearly, $B$ is bounded if and only if the sequence $\{\beta(k) / \beta(k+1)\}_{k}$ is bounded. The hereditarily hypercyclicity of $B$ on $L^{p}(\beta)$ with respect to the entire sequence is characterized in [12, Theorem 2.7].

Theorem 4.1. Suppose the operator $B$ is bounded on $X=L^{p}(\beta)$. Then $B$ is supercyclic on $X$ if and only if

$$
\liminf _{n \rightarrow \infty} \max \{\beta(k-n) \beta(j+n):|j|,|k| \leq q\}=0
$$

for all $q \in \mathbb{N}$.

Proof. $(\Rightarrow)$ Suppose $B$ is supercyclic on $X$. For $q \in \mathbb{N}$, consider the nonzero vector $g=h=\sum_{|j| \leq q} f_{j}$. Denote $\alpha_{0}=\min \{\beta(k):|k| \leq q\}$. By Remark 1.5, there exist a vector $x=\sum_{j} \hat{x}(j) f_{j} \in L^{p}(\beta)$, an arbitrary large positive integer $n>2 q$ and $0 \neq \alpha \in \mathbb{C}$ such that

$$
\|x-g\|<\epsilon \text { and }\left\|\alpha B^{n} x-h\right\|<\epsilon .
$$

That is,

$$
\left\|\sum_{j} \hat{x}(j) f_{j}-\sum_{|j| \leq q} f_{j}\right\|<\epsilon \text { and }\left\|\alpha \sum_{j} \hat{x}(j) f_{j-n}-\sum_{|j| \leq q} f_{j}\right\|<\epsilon .
$$

From which it follows that

$$
\begin{gathered}
|\hat{x}(j)-1| \beta(j)<\epsilon \Rightarrow|\hat{x}(j)|>1-\frac{\epsilon}{\alpha_{0}} ; \text { for }|j| \leq q . \\
|\hat{x}(j)| \beta(j)<\epsilon ; \text { for }|j|>q . \\
|\alpha \hat{x}(j+n)-1| \beta(j)<\epsilon \Rightarrow|\alpha \hat{x}(j+n)|>1-\frac{\epsilon}{\alpha_{0}} ; \text { for }|j| \leq q . \\
|\alpha \hat{x}(j+n)| \beta(j)<\epsilon ; \text { for }|j|>q .
\end{gathered}
$$

For $|k|,|j| \leq q$, by relations (4.2) and (4.5), it follows that

$$
\beta(k-n)<\frac{\epsilon}{\alpha|\hat{x}(k-n+n)|}=\frac{\epsilon}{\alpha|\hat{x}(k)|}<\frac{\epsilon}{\alpha\left(1-\frac{\epsilon}{\alpha_{0}}\right)}=\frac{\alpha_{0} \epsilon}{\alpha\left(\alpha_{0}-\epsilon\right)} ;
$$

and by relations (4.3) and (4.4), it follows that

$$
\beta(j+n)<\frac{\epsilon}{\hat{x}(j+n)}<\frac{\alpha \epsilon}{1-\frac{\epsilon}{\alpha_{0}}}=\frac{\alpha \alpha_{0} \epsilon}{\alpha_{0}-\epsilon} .
$$

Consequently, for all $|k|,|j| \leq q, n>2 q$, we have that

$$
\beta(k-n) \beta(j+n)<\frac{\alpha_{0}^{2} \epsilon^{2}}{\left(\alpha_{0}-\epsilon\right)^{2}} .
$$

Since $\epsilon$ is an arbitrary positive element, thus (4.1) follows.

$(\Leftarrow)$ Suppose $(4.1)$ holds. So there exists a sequence $\left\{n_{r}\right\}$ such that

$$
\lim _{r \rightarrow \infty} \max \left\{\beta\left(k-n_{r}\right) \beta\left(j+n_{r}\right):|j|,|k| \leq q\right\}=0 .
$$

Theorem 1.4 will be used to show the supercyclicity of $B$ on $X$. For $m \in \mathbb{N}$, let $X_{0}=Y_{0}:=\operatorname{span}\left\{f_{j}:|j| \leq m\right\}$ be the dense sets of $X$. Define $S: Y_{0} \rightarrow X$ 
as $S f_{j}=f_{j+1}$. It is obvious that $B S z=z$ for all $z \in Y_{0}$. On the other hand, if $y=\sum_{|j| \leq m} \hat{y}(j) f_{j} \in X_{0}$ and $z=\sum_{|h| \leq m} \hat{z}(h) f_{h} \in Y_{0}$, we obtain that

$$
\begin{aligned}
& \left\|B^{n_{r}} y\right\|\left\|S^{-n_{r}} z\right\|=\left\|\sum_{|j| \leq m} \hat{y}(j) f_{j-n_{r}}\right\|\left\|\sum_{|h| \leq m} \hat{z}(h) f_{h+n_{r}}\right\| \\
= & \left(\sum_{|j| \leq m}|\hat{y}(j)|^{p} \beta\left(j-n_{r}\right)^{p}\right)\left(\sum_{|h| \leq m}|\hat{z}(h)|^{p} \beta\left(h+n_{r}\right)^{p}\right) \\
= & \left(\sum_{|j| \leq m}|\hat{y}(j)|^{p} \beta(j)^{p} \frac{\beta\left(j-n_{r}\right)^{p}}{\beta(j)^{p}}\right)\left(\sum_{|h| \leq m}|\hat{z}(h)|^{p} \beta(h)^{p} \frac{\beta\left(h+n_{r}\right)^{p}}{\beta(h)^{p}}\right) \\
\leq & \sup _{|j| \leq m}\left(\frac{\beta\left(j-n_{r}\right)^{p}}{\beta(j)^{p}}\right) \sup _{|h| \leq m}\left(\frac{\beta\left(h+n_{r}\right)^{p}}{\beta(h)^{p}}\right)\|y\|\|z\| \\
\leq & \sup _{|j| \leq m}\left(\frac{\beta\left(j-n_{r}\right)^{p}}{\alpha_{0}^{p}}\right) \sup _{|h| \leq m}\left(\frac{\beta\left(h+n_{r}\right)^{p}}{\alpha_{0}^{p}}\right)\|y\|\|z\| .
\end{aligned}
$$

From the above inequality and (4.6), it follows that

$$
\lim _{r \rightarrow 1}\left\|B^{n_{r}} y\right\|\left\|S^{-n_{r}} z\right\|=0 \text { for } y \in Y_{0}, z \in Z_{0} .
$$

This completes the proof.

\section{Supercyclicity of weighted pseudo-shift on $F$-spaces}

F-spaces. F-spaces are kinds of completely metrisable topological vector spaces. It will be convenient to assume that the topology of an F-space is induced by an $F$-norm, which is always possible. We refer the readers to Chapter 2 in the book [6] by Grosse-Erdmann and Manguillot.

A sequence space is a (linear) subspace of the space $w=w(\mathbb{N})=\mathbb{K}^{\mathbb{N}}$ of all scalar sequences. We also consider bilateral sequence spaces, that is, subspaces of $w(\mathbb{Z})=\mathbb{K}^{\mathbb{Z}}$. In this section, we allow arbitrary countably infinite sets $I$ as index sets. Then a sequence space over $I$ is a subspace of the space $w(I)=\mathbb{K}^{I}$ of all scalar families $\left(x_{i}\right)_{i \in I}$. The space $w(I)$ is endowed with its natural product topology. By $e_{i}(i \in I)$ we denote the canonical unit vectors $e_{i}=\left(\delta_{i, k}\right)_{k \in I}$.

A topological sequence space $X$ over $I$ is a sequence space over $I$ that is endowed with a linear topology in such a way that the inclusion mapping $X \hookrightarrow w(I)$ is continuous or, equivalently, that every coordinate functional $f_{i}$ : $X \rightarrow \mathbb{K},\left(x_{k}\right)_{k \in I} \rightarrow x_{i}(i \in I)$, is continuous.

The family $\left(e_{i}\right)_{i \in I}$ of unit vectors is called an $M$-basis in a topological sequence space $X$ over $I$ if $\operatorname{span}\left\{e_{i}: i \in I\right\}$ is a dense subspace of $X$. We shall call $\left(e_{i}\right)_{i \in I}$ an OP-basis if it is an M-basis and if the family of coordinate projections $x \rightarrow x_{i} e_{i}(i \in I)$ on $X$ is equicontinuous. Now suppose that $X$ is an F-sequence space. Then if $\left(e_{n}\right)_{n \in \mathbb{N}}$ is a basis in $X$ it is also an OP-basis, and the converse is true under the stronger assumption that the sequence of partial 
sum operators $x \rightarrow \sum_{k=1}^{n} x_{k} e_{k}(n \in \mathbb{N})$ is equicontinuous. We refer the readers to $[5$, p. 50] for an example.

Definition 5.1. Let $X$ and $Y$ be topological sequence spaces over $I$ and $J$, respectively. Then a continuous linear operator $T: X \rightarrow Y$ is called a weighted pseudo-shift if there is a sequence $\left(b_{j}\right)_{j \in J}$ of nonzero scalars and injective mapping $\varphi: J \rightarrow I$ such that

$$
T\left(x_{i}\right)_{i \in I}=\left(b_{j} x_{\varphi(j)}\right)_{j \in J}
$$

for $\left(x_{i}\right) \in X$. We then write $T=T_{b, \varphi}$, and $\left(b_{j}\right)_{j \in J}$ is called the weight sequence.

We note that if $X$ and $Y$ are F-sequence spaces, by a standard argument using the closed graph theorem, the continuity of $T$ is automatic once it is known that $T$ maps each sequence from $X$ into $Y$. As we all know, a bilateral weighted backward or forward shift $T$ with some sequence $\left(a_{n}\right)_{n \in \mathbb{Z}}$ of nonzero scalars is defined by

$$
T\left(x_{n}\right)_{n \in \mathbb{Z}}=\left(a_{n+1} x_{n+1}\right)_{n \in \mathbb{Z}}
$$

or

$$
T\left(x_{n}\right)_{n \in \mathbb{Z}}=\left(a_{n-1} x_{n-1}\right)_{n \in \mathbb{Z}},
$$

respectively. The sequence $\left(a_{n}\right)_{n \in \mathbb{Z}}$ is called the weight sequence.

The unilateral weighted backward and forward shifts are defined analogously on topological sequence spaces over $\mathbb{N}$, where we set $a_{0}=x_{0}=0$. Thus from the definition, we have that every unilateral or bilateral weighted backward shift is a weighted pseudo-shift with $b_{n}=a_{n+1}$ and $\varphi(n)=n+1$, and every bilateral weighted forward shift is a weighted pseudo-shift with $b_{n}=a_{n-1}$ and $\varphi(n)=n-1$. In contrast, unilateral weighed forward shifts are never pseudoshifts due to their definition in the first component.

In order to describe the action of a pseudo-shift on the sequences $e_{i}(i \in I)$ we need to consider the inverse $\psi=\varphi^{-1}: \varphi(J) \rightarrow J$ of the mapping $\varphi: J \rightarrow I$. In addition we set $b_{\psi(i)}=0$ and $e_{\psi(i)}=0$ if $i \in I \backslash \varphi(J)$, that is, if $\psi(i)$ is "undefined". With the above definitions we have, for all $i \in I$,

$$
T_{b, \varphi} e_{i}=b_{\psi(i)} e_{\psi(i)}
$$

Definition 5.2. A sequence $\left(\varphi_{n}\right)_{n \in \mathbb{N}_{0}}$ of mappings $\varphi_{n}: J \rightarrow I$ is called a run-away sequence if for each pair of finite subsets $I_{0} \subset I$ and $J_{0} \subset J$, there exists an $n_{0} \in \mathbb{N}_{0}$ such that, for every $n \geq n_{0}, \varphi_{n}\left(J_{0}\right) \cap I_{0}=\emptyset$.

Let $T=T_{b, \varphi}: X \rightarrow X$ be a weighted pseudo-shift. In this section, we characterize the supercyclicity of $T$, which is a generalization of [5]. We first note that each $T^{n}\left(n \in \mathbb{N}_{0}\right)$ is a weighted pseudo-shift. That is,

$$
T^{n}\left(x_{i}\right)_{i \in I}=\left(b_{n, i} x_{\varphi_{n}(i)}\right)_{i \in I},
$$

where

$$
\varphi_{n}(i):=\varphi^{n}(i)=(\varphi \circ \varphi \circ \cdots \circ \varphi)(i) \quad(n \text {-fold })
$$




$$
b_{n, i}:=b_{i} b_{\varphi(i)} \ldots b_{\varphi^{n-1}(i)}=\prod_{v=0}^{n-1} b_{\varphi^{v}(i)} .
$$

Moreover, we add that $b_{\psi^{n}(i)}=0$ and $e_{\psi^{n}(i)}=0$ whenever $\psi^{n}(i)$ is "undefined".

Theorem 5.3. Let $X$ be an F-sequence space over $I$ in which $\left(e_{i}\right)_{i \in I}$ is an OP-basis. Let $T=T_{b, \varphi}: X \rightarrow X$ be a weighted pseudo-shift. If $\left(\varphi_{n}\right)$ is a run-away sequence, then the following assertions are equivalent:

(a) $T$ has a dense set of supercyclic vectors;

(b) there exists an increasing sequence $\left(n_{k}\right)$ of positive integers such that, for every $i, j \in I$,

$$
\left\|\left(\prod_{v=0}^{n_{k}-1} b_{\varphi^{v}(j)}\right)^{-1} e_{\varphi^{n_{k}(j)}}\right\|\left\|\left(\prod_{v=1}^{n_{k}} b_{\psi^{v}(i)}\right) e_{\psi^{n_{k}(i)}}\right\| \rightarrow 0,
$$

in $X$ as $k \rightarrow \infty$.

Proof. (b) $\Rightarrow(\mathrm{a})$. Suppose (b) holds. We set $X_{0}=Y_{0}=\operatorname{span}\left\{e_{i}, i \in I\right\}$, which are dense sets of $X$, since $\left(e_{i}\right)_{i \in I}$ is an OP-basis, and we define linear mappings $S_{n}: Y_{0} \rightarrow X$ be

$$
S_{n} e_{j}=\left(\prod_{v=0}^{n-1} b_{\varphi^{v}(j)}\right)^{-1} e_{\varphi^{n}(j)} \quad\left(n \in \mathbb{N}_{0}, j \in I\right) .
$$

Since

$$
T^{n} e_{i}=\left(\prod_{v=1}^{n} b_{\psi^{v}(i)}\right) e_{\psi^{n}(i)}\left(n \in \mathbb{N}_{0}, i \in I\right),
$$

and $T^{n} S_{n} e_{j}=e_{j}$ for $n \in \mathbb{N}_{0}, j \in I$. Moreover, by (5.1) it follows that

$$
\begin{aligned}
\lim _{k \rightarrow \infty}\left\|T^{n_{k}} e_{i}\right\|\left\|S_{n_{k}} e_{j}\right\| & =\lim _{k \rightarrow \infty}\left\|\left(\prod_{v=0}^{n_{k}-1} b_{\varphi^{v}(j)}\right)^{-1} e_{\varphi^{n_{k}(j)}}\right\|\left\|\left(\prod_{v=1}^{n_{k}} b_{\psi^{v}(i)}\right) e_{\psi^{n_{k}(i)}}\right\| \\
& =0 .
\end{aligned}
$$

By Theorem 1.4, T satisfies the Supercyclicity Criterion. By remark 1.5 it follows (a).

$(\mathrm{a}) \Rightarrow(\mathrm{b})$. Assume (a) holds. It suffices to show that for every pair of finite subsets $I_{0}$ and $J_{0}$ of $I$, for any $\epsilon>0$ and every $N \in \mathbb{N}$ there exists an $n>N$ such that

$$
\left\|\left(\prod_{v=0}^{n-1} b_{\varphi^{v}(j)}\right)^{-1} e_{\varphi^{n}(j)}\right\|\left\|\left(\prod_{v=1}^{n} b_{\psi^{v}(i)}\right) e_{\psi^{n}(i)}\right\|<\epsilon \text { in } X,
$$

for $i \in I_{0}$ and $j \in J_{0}$ where $\|\cdot\|$ denotes the $F$-norm in $X$. To see this we fix enumerations $\left(i_{k}\right)$ of $I$, and set $I_{k}=\left\{i_{1}, \ldots, i_{k}\right\}$ and $J_{k}=\left\{j_{1}, \ldots, j_{k}\right\}$, where $i_{m}, j_{m} \in I$ for $m=1, \ldots, k$. Next we define inductively an increasing sequence $\left(n_{k}\right)$ of positive integers by letting $n_{k}$ be a number $n$ satisfying (5.2) 
for $I_{0}=I_{k}, J_{0}=J_{k}, \epsilon=1 / k$ and $N=n_{k-1}$, where we set $n_{0}=0$. It is clear that the sequence $\left(n_{k}\right)$ satisfies (5.2), so that condition (b) holds.

We therefore have to prove (5.2) under the assumption of (a). Let $\epsilon>0$, finite subsets $I_{0} \subset I, J_{0} \subset J$, and $N \in \mathbb{N}$ be given. By the equicontinuity of the coordinate projections in $X$, for any $x \in X$, there is some $\delta>0$ such that

$$
\left\|x_{i} e_{i}\right\|<\epsilon / 2 \text { for } i \in I \text {, if }\|x\|<\delta .
$$

From (a), there exists $x \in X, 0 \neq \alpha \in \mathbb{C}$ and $N \in \mathbb{N}_{0}, n>N$, with

$$
\left\|x-\sum_{i \in I_{0}} e_{i}\right\|<\delta \text { and }\left\|\alpha T^{n} x-\sum_{j \in J_{0}} e_{j}\right\|<\delta .
$$

By continuous inclusion of $X$ into $w(I)$, we can in addition obtain that

$$
\sup _{i \in I_{0}}\left|\alpha x_{i}-1\right| \leq 1 / 2 \text { and } \sup _{j \in J_{0}}\left|y_{j}-1\right| \leq 1 / 2,
$$

where $y:=T^{n} x$. Since $\left(\varphi_{n}\right)$ is a run-away sequence we can assume that

$$
\varphi_{n}\left(J_{0}\right) \cap I_{0}=\emptyset .
$$

By (5.3), the first inequality in (5.4) implies that

$$
\left\|x_{i} e_{i}\right\|<\epsilon / 2 \text { if } i \in I \backslash I_{0} .
$$

hence by (5.6) we have that

$$
\left\|x_{\varphi_{n}(j)} e_{\varphi_{n}(j)}\right\|<\epsilon / 2 \text { for } j \in J_{0} .
$$

By the second inequality in (5.5) we have, for $j \in J_{0}$,

$$
\left|\left(\prod_{v=0}^{n-1} b_{\varphi^{v}(j)}\right) x_{\varphi_{n}(j)}-1\right| \leq 1 / 2,
$$

hence $x_{\varphi_{n}(j)} \neq 0$ and

$$
\left|\frac{1}{\left(\prod_{v=0}^{n-1} b_{\varphi^{v}(j)}\right) x_{\varphi_{n}(j)}}-1\right| \leq 1 .
$$

Now, (5.7) and (5.8) imply that, for $j \in J$,

$$
\begin{aligned}
& \left\|\left(\prod_{v=0}^{n-1} b_{\varphi^{v}(j)}\right)^{-1} e_{\varphi_{n}(j)}\right\|=\left\|\frac{1}{\left(\prod_{v=0}^{n-1} b_{\varphi^{v}(j)}\right) x_{\varphi_{n}(j)}} x_{\varphi_{n}(j)} e_{\varphi_{n}(j)}\right\| \\
\leq & \left\|x_{\varphi_{n}(j)} e_{\varphi_{n}(j)}\right\|+\left\|\left(\frac{1}{\left(\prod_{v=0}^{n-1} b_{\varphi^{v}(j)}\right) x_{\varphi_{n}(j)}}-1\right) x_{\varphi_{n}(j)} e_{\varphi_{n}(j)}\right\| \\
\leq & 2\left\|x_{\varphi_{n}(j)} e_{\varphi_{n}(j)}\right\|<\varepsilon,
\end{aligned}
$$

where we have used properties of $F$-norms. Deduce from (5.6) and the definition of the $\psi_{n}$ that

$$
\psi_{n}\left(I_{0} \cap \varphi_{n}(J)\right) \cap J_{0}=\emptyset .
$$


By (5.3), the second inequality in (5.4) implies that

$$
\left\|\alpha\left(\prod_{v=0}^{n-1} b_{\varphi^{v}(j)}\right) x_{\varphi_{n}(j)} e_{j}\right\|<\epsilon / 2 \text { if } j \in I \backslash J_{0} .
$$

Hence by (5.10) it follows that

$$
\left\|\alpha\left(\prod_{v=1}^{n} b_{\psi^{v}(i)}\right) x_{i} e_{\psi_{n}(i)}\right\|<\epsilon / 2 \text { for } i \in I_{0} ;
$$

note that $e_{\psi_{n}(i)}=0$ if $i \in I \backslash \varphi_{n}(J)$. By the first inequality in (5.5) we have that

$$
\left|\alpha x_{i}\right| \geq 1 / 2 \text { for } i \in I_{0},
$$

in particular $x_{i} \neq 0$. Now, (5.11) and (5.12) imply that

$$
\begin{aligned}
\left\|\left(\prod_{v=1}^{n} b_{\psi^{v}(i)}\right) e_{\psi_{n}(i)}\right\| & =\left\|\frac{1}{2 \alpha x_{i}} 2 \alpha\left(\prod_{v=1}^{n} b_{\psi^{v}(i)}\right) x_{i} e_{\psi_{n}(i)}\right\| \\
& \leq\left\|2\left(\prod_{v=1}^{n} b_{\psi^{v}(i)}\right) x_{i} e_{\psi_{n}(i)}\right\|<\epsilon
\end{aligned}
$$

for all $i \in I_{0}$, where we have used properties of $F$-norms. This completes the proof.

Remark 5.4. (1) The condition that $\left(\varphi_{n}\right)$ be a run-away sequence is clearly not necessary for the supercyclicity.

(2) As the proof shows, for the implication $(\mathrm{b}) \Rightarrow(\mathrm{a})$ it suffices that $\left(e_{i}\right)_{i \in I}$ is a $M$-basis in $X$, while for the implication $(\mathrm{a}) \Rightarrow(\mathrm{b})$ we need only assume the equicontinuity of the coordinate projections in $X$.

Theorem 5.5. Let $X$ be an F-sequence space over $I$ in which $\left(e_{i}\right)_{i \in I}$ is an $O P$ basis. Let $T=T_{b, \varphi}: X \rightarrow X$ be a weighted pseudo-shift. Then the following assertions are equivalent:

(a) T has a dense set of supercyclic vectors;

(b) (i) the mapping $\varphi: I \rightarrow I$ has no periodic points;

(ii) there exists an increasing sequence $\left(n_{k}\right)$ of positive integers such that, for every $i, j \in I$,

$$
\left\|\left(\prod_{v=0}^{n_{k}-1} b_{\varphi^{v}(j)}\right)^{-1} e_{\varphi^{n}(j)}\right\|\left\|\left(\prod_{v=1}^{n_{k}} b_{\psi^{v}(i)}\right) e_{\psi^{n_{k}(i)}}\right\| \rightarrow 0
$$

in $X$ as $k \rightarrow \infty$.

Proof. First assume that $\varphi$ has a periodic point, that is, $\varphi^{N}(i)=i$ for some $i \in I$ and $N \in \mathbb{N}$. Then for every $\alpha \neq 0$, the entry of $\alpha T^{n} x$ at the position $i$ is

$$
\alpha\left(\prod_{v=0}^{n-1} \beta_{v}\right) \zeta_{n} \text { with } \beta_{v}=b_{\varphi^{v}(i)} \text { and } \zeta_{n}=x_{\varphi^{n}(i)} .
$$


It is obvious that both $\left(\beta_{v}\right)_{v}$ and $\left(\zeta_{n}\right)_{n}$ are periodic sequences we see that for no $x \in X$ can these entries form a dense set in $\mathbb{K}$ as $n$ varies. Since $X$ contains $e_{i}$ and is continuously included in $\mathbb{K}^{I}$ this shows that $\left\{\alpha T^{n} x: n \in \mathbb{N}_{0}\right\}$ can not be dense in $X$ for any element $x$, hence that $T$ is not supercyclic.

Thus if $\varphi$ has no periodic points, then for every finite subset $I_{0}$ of $I$ and any $i \in I$ there is an $n_{0} \in \mathbb{N}$ with $\varphi^{n}(i) \in I \backslash I_{0}$ for $n \geq n_{0}$. This shows that $\left(\varphi^{n}\right)$ is a run-away sequence. Thus we can apply Theorem 5.3 to obtain the present theorem.

Acknowledgements. The authors would like to thank the referee for useful comments and suggestions which improved the presentation of this paper.

\section{References}

[1] F. Bayart and E. Matheron, Dynamics of Linear Operators, Camberidge University Press, 2009.

[2] J. Bès, Hereditarily hypercyclic operators, J. Funct. Anal. 167 (1999), no. 1, 94-112.

[3] R. Y. Chen and Z. H. Zhou, Hypercyclicity of weighted composition operators on the unit ball of $\mathbb{C}^{N}$, J. Korean Math. Soc. 48 (2011), no. 5, 969-984.

[4] G. Godefroy and J. H. Shapiro, Operators with dense, invariant, cyclic vector manifolds. J. Funct. Anal. 98 (1991), no. 2, 229-269.

[5] K. G. Grosse-Erdmann, Hypercyclic and chaotic weighted shifts, Studia Math. 139 (2000), no. 1, 47-68.

[6] K. G. Grosse-Erdmann and A. P. Manguillot, Linear Chaos, Springer, New York, 2011.

[7] M. Hazarika and S. C. Arora, Hypercyclic operator weighted shifts, Bull. Korean Math. Soc. 41 (2004), no. 4, 589-598.

[8] C. Kitai, Invariant closed sets for linear operators, Phd thesis, Univ. of Toronto, 1982.

[9] S. Rolewicz, On orbits of elements, Studia Math. 32 (1969), 17-22.

[10] H. N. Salas, Hypercyclic weighted shifts, Trans. Amer. Math. Soc. 347 (1995), no. 3, 993-1004.

[11] _ Supercyclicity and weighted shifts, Studia Math. 135 (1999), no. 1, 55-74.

[12] B. Yousefi and A. Farrokhinia, On the hereditarily hypercyclic operators, J. Korean Math. Soc. 43 (2006), no. 6, 1219-1229.

Yu-Xia Liang

Department of Mathematics

TIANJIN Universit

Tianjin 300072, P. R. China

E-mail address: liangyx1986@126.com

Ze-Hua Zhou

Department of Mathematics

TianJin Universit

Tianjin 300072, P. R. China

E-mail address: zehuazhoumath@aliyun.com;zhzhou@tju.edu.cn 